\title{
ON DISCREPANCY THEOREMS WITH APPLICATIONS TO APPROXIMATION THEORY
}

\author{
HANS-PETER BLATT \\ Mathematisch-Geographische Fakultät, Katholische Universität Eichstätt \\ Ostenstr. 26-28, D-85071 Eichstätt, Germany
}

We give an overview on discrepancy theorems based on bounds of the logarithmic potential of signed measures. The results generalize well-known results of P. Erdős and P. Turán on the distribution of zeros of polynomials. Besides of new estimates for the zeros of orthogonal polynomials, we give further applications to approximation theory concerning the distribution of Fekete points, extreme points and zeros of polynomials of best uniform approximation.

1. Introduction. In many situations in approximation theory the distribution of points with respect to some set is of interest. For example, an important problem is the distribution of the points of alternation of the error function in polynomial Chebyshev approximation on a real interval. This problem was first considered by Kadec [10] and leads to estimates between the distribution of these points and the equilibrium measure of the interval. Walsh [20] considered interpolations points on the boundary of a set $E$ where the function $f$ has to be approximated by polynomials. Again the distribution of the interpolation points has to be related to the equilibrium distribution of $E$ to obtain sufficiently good approximations.

If we know only a bound on the Chebyshev norm of a polynomial on some set in the complex plane, the first result on the distribution of the zeros of this polynomial dates back to Erdős and Turán: Let $p \in \Pi_{n}$ be a monic polynomial where $\Pi_{n}$ denotes the set of all algebraic polynomials of degree at most $n$ and let $\tau$ be the normalized zero-counting measure of $p$, i.e.

$$
\tau(A):=\frac{\text { number of zeros of } p \text { in } A}{n}
$$

1991 Mathematics Subject Classification: 30C15, 41A10.

The paper is in final form and no version of it will be published elsewhere. 
where $A$ is any point set in $\mathbb{C}$ and the zeros are counted with their multiplicities.

If all zeros of $p$ are in the interval $[-1,1]$ then Erdös and Turán [6] proved for any subinterval $[a, b]$ of $[-1,1]$ that

$$
\left|\tau([a, b])-\frac{\beta-\alpha}{\pi}\right| \leq \frac{8}{\log 3} \sqrt{\log 2+\frac{\log \|p\|_{[-1,1]}}{n}}
$$

where $a=\cos \beta, b=\cos \alpha, 0 \leq \alpha \leq \beta \leq \pi$, and $\|p\|_{[-1,1]}$ denotes the Chebyshev norm of $p$ on $[-1,1]$. Note that

$$
\log 2+\frac{\log \|p\|_{[-1,1]}}{n} \geq \frac{\log 2}{n} .
$$

The corresponding result for the unit disk was also proved by Erdős and Turán [7]:

Let $p_{n}(z)=z^{n}+\ldots+a_{0} \in \Pi_{n}$ be a monic polynomial, $a_{0} \neq 0$, and let

$$
S_{\alpha, \beta}=\left\{r e^{i \varphi}: r \geq 0,0 \leq \alpha \leq \varphi \leq \beta \leq 2 \pi\right\}
$$

then

$$
\left|\tau\left(S_{\alpha, \beta}\right)-\frac{\beta-\alpha}{2 \pi}\right| \leq 16 \sqrt{\frac{\log \|p\|_{|z|=1}}{n}-\frac{\log \left|a_{0}\right|}{2 n}}
$$

where $\|p\|_{|z|=1}$ is the uniform norm of $p$ on the unit circle. Remember that

$$
\frac{\log \|p\|_{|z|=1}}{n}-\frac{\log \left|a_{0}\right|}{2 n} \geq\left|\frac{\log \left|a_{0}\right|}{2 n}\right|>0 \text {. }
$$

The main idea to generalize (1.2) resp. (1.3) to other situations can be found by rewriting the discriminant under the square root in a potential theoretical way. Therefore we need some basis facts from potential theory.

Let $E \subset \mathbb{C}$ be compact and $\mathcal{M}(E)$ denote the collection of all positive unit Borel measures supported on $E$. For $\sigma \in \mathcal{M}(E)$ the energy of $\sigma$ is defined by the formula

$$
I[\sigma]:=\int U^{\sigma}(z) d \sigma(z)
$$

where

$$
U^{\sigma}(z):=\int \log \left(\frac{1}{|z-t|}\right) d \sigma(t)
$$

is the logarithmic potential of $\sigma$. If

$$
W(E):=\inf _{\sigma \in \mathcal{M}(E)} I(\sigma)
$$

then the (logarithmic) capacity of $E$ is defined by

$$
\operatorname{cap}(E):=\exp (-W(E))
$$

If $\operatorname{cap}(E)>0$ then there exists (cf. [19], Chapter III) a unique measure $\mu_{E} \in$ $\mathcal{M}(E)$ such that

$$
I\left[\mu_{E}\right]=W(E) .
$$


The measure $\mu_{E}$ is called the equilibrium measure of $E$. Let $G$ be the Green's function of the unbounded component $\Omega(E)$ of $\mathbb{C} \cup\{\infty\} \backslash E$. If $\operatorname{cap}(E)>0$, then $G$ is connected with the logarithmic potential of $\mu_{E}$ by

$$
U^{\mu_{E}}(z)=-G(z)-\log \operatorname{cap}(E), \quad z \in \Omega(E)
$$

([19], Theorem III. 37, p. 82). $G$ tends to zero at all regular points of the boundary of $\Omega(E)$. In particular, if $E$ is a Jordan curve or a Jordan arc then $G$ can be continuously extended to $E$ such that $G(z)=0$ for $z \in E$ and (1.8) holds for $z \in E$.

We denote by

$$
\Gamma_{\alpha}:=\{z \in \Omega(E): G(z)=\log \alpha\}, \quad \alpha>1,
$$

the level lines of the Green function $G(z)$ and set, for $\alpha>1$,

$$
E_{\alpha}:=\{z \in \Omega(E): 0<G(z) \leq \log \alpha\} \cup E .
$$

Every level line $\Gamma_{\alpha}$ is analytic at each point $z$ where $\operatorname{grad} G(z) \neq 0$. Moreover, $\Gamma_{\alpha}$ consists of a finite number of Jordan curves which are naturally exterior except for a finite number of critical points $([20])$ where $\operatorname{grad} G(z)=0$.

2. Erdős-Turán-type theorems: Estimates by one-side bounds of $U^{\mu}-U^{\tau}$. The main idea for generalizing (1.2) to more general situations is to rewrite a bound for the Chebyshev norm of $p \in \Pi_{n}$,

$$
\|p\|_{[-1,1]} \leq \frac{A_{n}}{2^{n}}=A_{n} \operatorname{cap}(E)^{n}
$$

(where $E=[-1,1]$ ) in a potential theoretical way. Hence $(2.1)$ leads to

$$
\frac{1}{n} \log |p(z)|-\log \operatorname{cap}(E)-G(z) \leq \frac{\log A_{n}}{n}
$$

for $z \in E$ (cf. [2]) or

$$
U^{\mu}(z)-U^{\tau}(z) \leq \frac{\log A_{n}}{n}, \quad z \in E,
$$

where $\tau$ is the normalized zero-counting measure of $p$ and $\mu=\mu_{E}$ is the equilibrium measure of $E=[-1,1]$. Since $U^{\mu}-U^{\tau}$ is subharmonic, the maximum principle yields

$$
\left(U^{\mu}-U^{\tau}\right)(z) \leq \frac{\log A_{n}}{n}=: \varepsilon, \quad z \in \mathbb{C}
$$

and we get by $(1.2)$ and $(2.1)$

$$
\left|\tau([a, b])-\frac{\beta-\alpha}{\pi}\right|=|(\tau-\mu)([a, b])| \leq \frac{8}{\log 3} \sqrt{\varepsilon} .
$$

Hence, we have got an estimate for the modulus of $\mu-\tau$ on subintervals of $E=[-1,1]$ by a one-sided bound for the logarithmic potential $U^{\mu}-U^{\tau}$.

Instead of $E=[-1,1]$, let $E$ be a Jordan arc of class $C^{2+}$ and let $\tau$ be again the normalized zero-counting measure of a polynomial $p$. Let us now introduce 
the so-called Green lines (see [12]). These are orthogonal trajectories of the family of level lines of $G(z)$ and any $z \in E$ is the endpoint of a unique Green line $L(z)$. For any subarc $J$ of $E$ we define

$$
S_{\alpha}^{+}(J)=\left(\bigcup_{z \in J} L(z)\right) \cap E_{\alpha}
$$

Then the generalization of the Erdős-Turán estimate (2.4) is the following.

TheOREM 1 [2]. Let $E$ be a Jordan arc of class $C^{2+}, c_{0}>0$ fixed. Then there exists a constant $C>0$, depending on $E$ and $c_{0}$, such that for all subarcs $J$ of $E$ and all $\alpha \geq 1+c_{0} \sqrt{\varepsilon}$ the estimate

$$
\mid(\mu-\tau)\left(S_{\alpha}^{+}(J) \mid \leq C \sqrt{\varepsilon}\right.
$$

holds where $\tau$ is the normalized zero-counting measure of a polynomial, $\mu$ the equilibrium measure of $E$ and

$$
\varepsilon:=\sup \left\{U^{\mu-\tau}(z): z \in \mathbb{C}\right\} .
$$

We remark that $S_{\alpha}^{+}(J)$ is a neighbourhood of each interior point of the Jordan arc $J$ and we have not any more the condition that $\tau$ is supported by $E$.

Next, we consider the generalization of (1.2) to a Jordan curve $E$ instead of the unit circle: Let $E$ be Jordan curve of class $C^{2+}$, and let $S_{\alpha}^{+}(J)$ be defined as above, $\alpha>1$. Moreover, we fix a point $z_{0}$ in the interior of $E$. Let $G_{0}(z)=G_{0}\left(z, z_{0}\right)$ be the Green function of the region $F=\operatorname{int}(E)$ with pole at $z_{0}$. For $z \in E$ we denote by $L_{0}(Z)$ the Green line of $G_{0}(z)$ ending in $z \in E$, and let

$$
F_{\alpha}:=\left\{z \in F: 0<G_{0}(z) \leq \log \alpha\right\} \cup E
$$

for $\alpha>1$, and set for any subarc $J$ of $E$

$$
S_{\alpha}^{-}(J)=\left(\bigcup_{z \in J} L_{0}(z)\right) \cap F_{\sigma}
$$

and

$$
S_{\alpha}(J)=S_{\alpha}^{+}(J) \cup S_{\alpha}^{-}(J) .
$$

TheOREM $2[2]$. Let $E$ be a Jordan curve of class $C^{2+}, z_{0}$ a fixed point of the interior of $E, c_{0}>0$. Then there exists a constant $C>0$, depending on $E, z_{0}$ and $c_{0}$ such that for all subarc $J$ of $E$ and all $\alpha \geq 1+c_{0} \sqrt{U^{\tau-\mu}+2 \varepsilon}$

$$
\left|(\mu-\tau)\left(S_{\alpha}(J)\right)\right| \leq C \sqrt{U^{\tau-\mu}\left(z_{0}\right)+2 \varepsilon}
$$

where $\tau$ is the normalized zero-counting measure of a polynomial $p, \mu=\mu_{E}$ and

$$
\varepsilon:=\sup \left\{U^{\mu-\tau}(z): z \in \mathbb{C}\right\} .
$$

The estimate (1.2) of Erdős-Turán fits in this general framework. Namely, if $E$ is the unit circle we choose $z_{0}$ to be the point $z_{0}=0$. Then

$$
G_{0}(z)=-\log |z|, \quad|z|<1
$$

and 


$$
U^{\tau-\mu}(z)=U^{\tau}(z)-U^{\mu}(z)=-\frac{\log \left|p_{n}(z)\right|}{n}
$$

since $U^{\mu}(z)=0$ for $|z| \leq 1$. Hence,

$$
\begin{gathered}
U^{\tau-\mu}\left(z_{0}\right)=U^{\tau-\mu}(0)=-\frac{\log \left|a_{0}\right|}{n}, \\
\varepsilon=\sup \left\{U^{\mu-\tau}(z): z \in \mathbb{C}\right\}=\frac{\log \|p\|_{|z|=1}}{n}
\end{gathered}
$$

and we obtain immediately from (2.11) the former inequality (1.3) of Erdős and Turán.

We remark that in [2] Theorems 1 and 2 were proved for the more general situation that $E$ can be a general regular set, but $J$ has to be a subarc of a Jordan curve (resp. Jordan arc). Recently, these theorems were generalized by Grothmann [9] for the case that $\tau$ is any probability measure satisfying the one-side bound (2.7), resp. (2.12), between the logarithmic potentials of the measure $\tau$ and the equilibrium distribution of $E$. Moreover, Grothmann has given estimates in the case that $E$ is a Jordan curve (resp. Jordan arc) with corners. In his results the angles of the corners play a significant role. So far it is not known how sharp the results of Grothmann are. On the other hand the result of Theorem 1 is sharp. This was already pointed out by Erdös and Turán.

3. Estimates by two-sided bounds of $U^{\mu}-U^{\tau}$. The sharpness of the Erdős-Turán results for $E=[-1,1]$ can be shown by modified Jacobi polynomials having multiple zeros at the endpoints $([4])$, i.e. if we know only the norm of the polynomial

$$
p(x)=x^{n}+\ldots
$$

on $[-1,1]$ then no better estimates can be given besides of the constant $8 / \log 3$ in (1.2) (cf. [8], [14]). But if we assume that all zeros are "sufficiently simple" the estimate can be substantially improved. To be precise let

$$
\|p\|_{[-1,1]} \leq A_{n} \frac{1}{2^{n}}
$$

and let

$$
\left|p^{\prime}\left(x_{i}\right)\right| \geq \frac{1}{B_{n}} \frac{1}{2^{n}}
$$

for all zeros $x_{i}$ of $p, i=1, \ldots, n$.

THEOREM 3 [3]. Let $p$ be a monic polynomial of degree $n$ with simple zeros $x_{i}$ on $[-1,1]$ such that (3.1) and (3.2) hold. Moreover, let $\tau$ be the normalized zero-counting measure of $p$ and $\mu$ the equilibrium distribution of $[-1,1]$. Then there exists a constant $c>0$ such that for any subinterval $[a, b]$ of $[-1,1]$

$$
|(\mu-\tau)([a, b])| \leq c \log n \frac{\log C_{n}}{n}
$$

where $C_{n}=\max \left(A_{n}, B_{n}, n\right)$. 
V. Totik [18] has obtained better estimates in Theorem 3, namely

$$
|(\mu-\tau)([a, b])| \leq c \frac{\log C_{n}}{n} \log \left(\frac{n}{\log C_{n}}\right)
$$

if $\log C_{n} / n$ is less than a fixed constant less than 1 . Moreover, Totik could prove that (3.4) is sharp.

The proof of (3.3) in [3] is of function theoretical nature and uses essentially the fact that the conditions (3.1) and (3.2) lead to

$$
\left|U^{\tau-\mu}(z)\right| \leq c \frac{\log C_{n}}{n}, \quad|z| \geq 1+n^{-\kappa}
$$

where $\kappa>0$ and the constant $c$ may depend on $\kappa$ but is independent of $n$.

The starting point for generalizing Theorem 3 to a Jordan curve or arc $E$ is the two-sided bound (3.5) outside certain level lines of the Green function $G(z)$ of the exterior of $E$. Let us introduce the notion of discrepancy $D\left[\sigma^{+}-\sigma^{-}\right]$between two probability measures $\sigma^{+}$and $\sigma^{-}$, i.e.

$$
D\left[\sigma^{+}-\sigma^{-}\right]:=\sup \left|\left(\sigma^{+}-\sigma^{-}\right)(J)\right|
$$

where the supremum is taken over all subarc $J \subseteq E$. Now, the general discrepancy theorem only makes use of a bound

$$
\varepsilon(\alpha) \geq \max _{z \in \Gamma_{\alpha}}\left|U^{\sigma^{+}-\sigma^{-}}(z)\right|
$$

THEOREM 4 [5]. Let $E$ be a Jordan curve or a Jordan arc of class $C^{1+}, \sigma^{+}$and $\sigma^{-}$two probability measures on $E$. Moreover, let $M>0,0<\gamma \leq 1$ be constants such that for all subarc $J$ of $E$

$$
\sigma^{+}(J) \leq M\left(\int_{J} d s\right)^{\gamma}
$$

Then there exists a constant $c>0$ depending on $E, \mathcal{M}$ and $\gamma$ such that

$$
D\left[\sigma^{+}-\sigma^{-}\right] \leq c \varepsilon(\alpha) \log \frac{1}{\varepsilon(\alpha)}
$$

for all $\alpha$ with $\alpha \leq 1+\varepsilon(\alpha)^{1+1 / \gamma}$ and $\varepsilon(\alpha)<1 / e$.

\section{Applications}

(a) Zeros of Chebyshev polynomials. Let $E$ be a Jordan arc of class $C^{2+}$ and let $T_{n}(z)$ be the monic Chebyshev polynomial on $E$ of degree $n$. We denote by $Z_{n}(K)$ the number of zeros of $T_{n}(z)$ in $K$. Due to a result of Widom [21] there exists a constant $c>0$ such that

$$
\left\|T_{n}\right\|_{E} \leq c \operatorname{cap}(E)^{n} .
$$

Now, Theorem 1 yields (for any fixed $\alpha_{0}>0$ )

$$
\left|\frac{Z_{n}\left(S_{\alpha}^{+}(J)\right)}{n}-\mu_{E}\left(S_{\alpha}^{+}(J)\right)\right| \leq C / \sqrt{n}
$$


for all $\alpha \geq \alpha_{0}$ where $C$ is a constant depending on $E$ and $\alpha_{0}$.

(b) Zeros of polynomials of best uniform approximation. Let $E$ be a Jordan arc of class $C^{2+}$ and $f$ a continuous function on $E$ which is not infinitely often differentiable on the boundary of $E$. Let $p_{n}^{*}$ be the polynomial of best uniform approximation to $f$ on $E$ of degree $\leq n$. Again, $\tau_{n}^{*}$ denotes the normalized zero counting measure of $p_{n}^{*}$. Then by Theorem 1

$$
\mid\left(\tau_{n}^{*}-\mu_{E}\right)\left(S_{\alpha}^{+}(J) \mid \leq C \sqrt{\frac{\log n}{n}}\right.
$$

for any subarc $J \subset E$ and any $\alpha \geq \alpha_{0}>1$ where $C$ is a constant depending on $E$ and $\alpha_{0}$ (Blatt, Grothmann [2]).

If $E$ is a Jordan curve an analogous result is true (cf. [2], Corollary 4).

(c) Fekete points. Let $E$ be a Jordan arc or curve as section 3 and let $\mathcal{F}_{n}(E)$ be any $n$-point subset $S$ of $E$ for which the Vandermonde expression

$$
V(S):=\left\{\prod_{\substack{z, t \in S \\ z \neq t}}|z-t|\right\}^{1 / 2}
$$

is as large as possible. The points in $\mathcal{F}_{n}(E)$ are called Fekete points of $E$ and these points are related to the capacity by

$$
\lim _{n \rightarrow \infty} V\left(\mathcal{F}_{n}(E)\right)^{2 / n(n-1)}=\operatorname{cap}(E) .
$$

Let $\nu_{n}$ denote the measure that associates the mass $1 / n$ with each of the Fekete points in $\mathcal{F}_{n}(E)$ then

$$
D\left[\nu_{n}-\mu_{E}\right] \leq c \frac{(\log n)^{2}}{n}
$$

where $c$ is a constant dependent on $E$ (Blatt, Mhaskar [5]). Previous results are due to Kleiner [11] and Pommerenke [15], [16].

(d) Extreme points. Let $E$ be again a Jordan arc or Jordan curve of class $C^{1+}$. If $E$ is a Jordan arc then we set $K:=E$. In the case that $E$ is a Jordan curve we define $K$ as the closed region bounded by $E$. Next, we consider the approximation of a continuous function $f$ on $K$ which is analytic in the interior of $K$. Let $p_{n}^{*}$ be the best Chebyshev approximation to $f$ on $K$ by $\Pi_{n}$. The distribution of the points in the extreme point set

$$
A_{n}(f):=\left\{z \in E:\left|f(z)-p_{n}^{*}(z)\right|=\left\|f-p_{n}^{*}\right\|_{E}\right\}
$$

can be estimated at least for the Fekete points of this set. Let $\mathcal{F}_{n+2}\left(A_{n}(f)\right)$ be any $n+2$ point Fekete set of $A_{n}(f)$ and let $\nu_{n+2}$ be the measure that associates the mass $1 / n+2$ with each point of this set. Then Blatt, Saff and Totik proved in [1] that

$$
D\left[\nu_{n+2}-\mu_{E}\right] \leq c \frac{\log n}{\sqrt{n}}
$$


for infinitely many integers $n$ in the case of a Jordan curve $E$ of class $C^{1+}$. Now, Theorem 4 yields for infinitely many $n$

$$
D\left[\nu_{n+2}-\mu_{E}\right] \leq c \frac{(\log n)^{2}}{\sqrt{n}}
$$

if $E$ is either Jordan curve or a Jordan arc of class $C^{1+}([5])$, a result which is slightly weaker than (4.1), but is true for a Jordan arc too.

(e) Orthogonal polynomials. Let $E=[-1,1]$ and $\tau$ be a unit Borel measure with finite moments. Moreover, we assume that the support of $\tau$ is infinite. Then there exists a unique system of orthonormalized polynomials

$$
p_{n}(x)=\gamma_{n} x^{n}+\ldots, \quad \gamma_{n}>0
$$

such that

$$
\int_{-1}^{1} p_{n} p_{m} d \tau=\delta_{n, m}, \quad n, m=0,1, \ldots
$$

For each integer $n \geq 1$, the polynomials $p_{n}$ have $n$ simple zeros in $[-1,1]$. If the Radon-Nikodym derivative $\tau^{\prime}$ satisfies $\tau^{\prime} \geq \kappa>0$ on $[-1,1]$ then

$$
D\left[\nu_{n}-\mu_{E}\right] \leq c \frac{(\log n)^{2}}{n}
$$

where $\nu_{n}$ is the normalized zero-counting measure of the zeros of $p_{n}$ ([3]). More general results can be found in Blatt, Mhaskar [5] as well results on the distribution of the zeros of orthogonal polynomials on the unit circle.

\section{References}

[1] H.-P. Blatt, E. B. Saff and V. Totik, The distribution of extreme points in best complex polynomial approximation, Constr. Approx. 5 (1989), 357-370.

[2] H.-P. Blatt and R. Grothmann, Erdös-Turán theorems on a system of Jordan curves and arcs, ibid. 7 (1991), 19-47.

[3] H.-P. Blatt, On the distribution of simple zeros of polynomials, Approx. Theory 69 (1992), 250-268.

[4] -, Verteilung der Nullstellen von Polynomen auf Jordanbögen, in: P. Ganzinger (ed.), Informatik, Festschrift zum 60. Geburstag von G. Hotz, Teubner, Stuttgart, 1992.

[5] H.-P. Blatt and H. N. Mhaskar, A general discrepancy theorem, Ark. Mat., to appear.

[6] P. Erdős and P. Turán, On the uniformly dense distribution of certain sequences of points, Ann. Math. 41 (1940), 162-173.

[7] - - - On the uniformly dense distribution of certain sequences of points, ibid. 51 (1950), 105-119.

[8] T. Ganelius, Sequences of analytic functions and their zeros, Ark. Mat. 3 (1953), $1-50$.

[9] R. Grothmann, Interpolation points and zeros of polynomials in approximation theory, Habilitationsschrift, Kath. Universität Eichstätt, 1993

[10] M. I. Kadec, On the distribution of points of maximum deviation in the approximation of continuous functions by polynomials, Amer. Math. Soc. Transl. (2)26 (1963), 231234. 
[11] W. Kleiner, Sur l'approximation de la représentation conforme par la méthode des points extrémaux de M. Leja, Ann. Polon. Math. 14 (1964), 131-140.

[12] N. S. Landkof, Foundations of Modern Potential Theory, Springer, New York, 1972.

[13] H. N. Mhaskar, Some discrepancy theorems, in: Approximation Theory, Tampa, E. B Saff (ed.), Lecture Notes in Math. 1287, Springer, New York, 117-131.

[14] M. Mignotte, Remarque sur une question relative à des fonctions conjuguées, C. R. Acad. Sci. Paris, to appear.

[15] Ch. Pommerenke, Über die Verteilung der Fekete-Punkte, Math. Ann. 168 (1967), 111-127.

[16] —, Über die Verteilung der Fekete-Punkte II, ibid. 179 (1969), 212-218.

[17] P. Sjögren, Estimates of mass distributions from their potentials and energies, Ark. Mat. 10 (1972), 59-77.

[18] V. Totik, Distribution of simple zeros of polynomials, Acta Math. 170 (1993), 1-28.

[19] M. Tsuji, Potential Theory in Modern Function Theory, Chelsea, New York, 1950.

[20] J. L. Walsh, Interpolation and Approximation by Rational Functions in the Complex Domain, Amer. Math. Soc. Colloq. Publ. 20, 5th ed., Providence, 1969.

[21] H. Widom, Extremal polynomials associated with a system of curves in the complex plane, Adv. in Math. 3 (1969), 127-232. 